\title{
SYMPLECTIC FIBRATIONS AND RIEMANN-ROCH NUMBERS OF REDUCED SPACES
}

\author{
MARK HAMILTON AND LISA JEFFREY
}

\begin{abstract}
In this article we give formulas for the Riemann-Roch number of a symplectic quotient arising as the reduced space of a coadjoint orbit $\mathcal{O}_{\Lambda}$ (for $\Lambda \in \mathfrak{g}^{*}$ close to 0 ) as an evaluation of cohomology classes over the reduced space at 0 . This formula exhibits the dependence of the Riemann-Roch number on $\Lambda$. We also express the formula as a sum over the components of the fixed point set of the maximal torus. Our proof applies to Hamiltonian $G$-manifolds even if they do not have a compatible Kähler structure, using the definition of quantisation in terms of the Spin- $\mathbb{C}$ Dirac operator.
\end{abstract}

\section{INTRODUCTION}

Let $(M, \omega)$ be a compact symplectic manifold possessing a Hamiltonian action of a compact connected simply connected Lie group $G$, with moment map $\mu: M \rightarrow \mathfrak{g}^{*}$ (where $\mathfrak{g}$ is the Lie algebra of $G$ ). One can form the symplectic reduction

$$
M_{0}=\mu^{-1}(0) / G \text {, }
$$

or more generally

$$
M_{\Lambda}=\mu^{-1}\left(\mathcal{O}_{\Lambda}\right) / G
$$

for $\Lambda \in \mathfrak{g}$, where $\mathcal{O}_{\Lambda} \subseteq \mathfrak{g}^{*}$ is the orbit of $\Lambda$ under the coadjoint action.

We assume 0 is a regular value of $\mu$, and 0 is a regular value of $\mu_{\Lambda}: M \times \mathcal{O}_{\Lambda} \rightarrow \mathfrak{g}^{*}$ where $\mu_{\Lambda}(m, \xi)=\mu(m)-\xi$ for $m \in M$ and $\xi \in \mathcal{O}_{\Lambda}$. This is equivalent to assuming that $G$ acts with finite stabilizers on $\mu^{-1}(0)$ (resp. $\mu_{\Lambda}^{-1}(0)$ ) [8], so under this hypothesis $M_{0}$ and $M_{\Lambda}$ have at worst finite quotient singularities. We assume that $G$ acts freely on $\mu_{\Lambda}^{-1}(0)$ and $\mu^{-1}(0)$, so that $M_{\Lambda}$ is a smooth symplectic manifold. Denote the symplectic form on $M_{\Lambda}$ by $\omega_{\Lambda}$.

Let $L$ be a complex line bundle over $M_{\Lambda}$ with a connection whose curvature is equal to $\omega_{\Lambda}$, called a prequantum line bundle. If $M$ has a complex structure compatible with the symplectic structure (in other words $M$ is Kähler), then the quantisation of $M_{\Lambda}$ is defined as the virtual vector space

$$
\mathcal{Q}\left(L^{k}\right)=H^{0}\left(M, L^{k}\right)-H^{1}\left(M, L^{k}\right)+H^{2}\left(M, L^{k}\right)-\ldots
$$

where $H^{j}\left(M, L^{k}\right)$ is the $j$-th Dolbeault cohomology of $M$ with coefficients in $L^{k}$. (When $k$ is very large, all the $H^{j}\left(M, L^{k}\right)=0$ for $j \neq 0$, so the quantisation $\mathcal{Q}$ is simply a vector

Date: November 8, 2018.

MH was supported in part by OGSST and University of Toronto.

LJ was supported by a grant from NSERC.

This article makes up part of the Ph.D. thesis of the first author, under the supervision of the second author. 
space.) The dimension of the quantisation is given by the Riemann-Roch number. The formula for the Riemann-Roch number in terms of characteristic classes is given below at (3).

As has been observed by Duistermaat [5], Guillemin [7] and Vergne [21], even when $M$ is not Kähler (but is equipped only with an almost complex structure compatible with the symplectic structure - such almost complex structures always exist, as explained in the above references) one can still define the quantisation using an elliptic complex given by the Spin- $\mathbb{C}$ Dirac operator. In this more general situation, the dimension of the quantisation is still given by the Riemann-Roch number, the formula for which is still given in terms of characteristic classes by (3) below (see [5], Proposition 13.1). The quantisation using the spin- $\mathbb{C}$ Dirac operator has been extensively studied by Meinrenken 17, 18.

In this article we give formulas for the Riemann-Roch number of $L^{k}$ over the reduced space $M_{\Lambda}$ (for $\Lambda \in \mathfrak{g}^{*}$ close to 0 ) as an evaluation of cohomology classes over the reduced space $M_{0}$. This formula exhibits the dependence of the Riemann-Roch number on $\Lambda$. We also express the formula as a sum over the components of the fixed point set of the maximal torus. In Section 2.1 we give a simple proof for the Kähler case, which was suggested by the referee. In Section 2.2 we treat the non-Kähler case.

\section{Symplectic Fibrations}

It is a standard result (see for example [8]) that for $\Lambda$ in a neighbourhood of 0 in $\mathfrak{g}^{*}$, we have a fibration

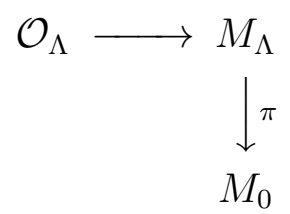

If $M$ is Kähler and the $G$ action preserves the Kähler structure, then $M_{\Lambda}$ and $M_{0}$ are also Kähler and (2) is a fibration of Kähler manifolds.

Let $L$ be a line bundle over $M_{\Lambda}$ with Chern character equal to $e^{\omega_{\Lambda}}$, (for example a prequantum line bundle) and let $k \in \mathbb{Z}$. The Riemann-Roch number of $L^{k}$ is then

$$
R R\left(M_{\Lambda}, L^{k}\right)=\int_{M_{\Lambda}} \operatorname{ch}\left(L^{k}\right) \operatorname{Td}\left(M_{\Lambda}\right)
$$

where $\operatorname{Td}\left(M_{\Lambda}\right)$ means $\operatorname{Td}\left(T M_{\Lambda}\right)$. The goal of this section is to express the Riemann-Roch number (3) as far as possible using terms defined on $M_{0}$.

Let

$$
\lambda=k \Lambda
$$

where $k$ is a positive integer. We require that $\lambda$ lie in the weight lattice $\Lambda^{W} \subset \mathfrak{t}^{*}$, which is the dual of the integer lattice $\Lambda^{I} \subset \mathfrak{t}$ (the kernel of the exponential map $\mathfrak{t} \rightarrow T$ ). We do not require that $\Lambda \in \Lambda^{W}$. 
2.1. The Kähler case. When $M_{0}$ and $M_{\Lambda}$ are Kähler, there is the following straightforward proof (which was pointed out by the referee). Let $V(\lambda)^{*}$ be the irreducible representation of $G$ with lowest weight $-\lambda$ (the dual of the irreducible representation with highest weight $\lambda$ ). By using the principal $G$-bundle $p_{0}: \mu^{-1}(0) \rightarrow M_{0}$, this representation yields a vector bundle $\mathcal{V}(\lambda)^{*}$ on $M_{0}$. We introduce a line bundle $L_{0}$ on $M_{0}$ for which $c_{1}\left(L_{0}\right)=\left[\omega_{0}\right]$, where $\omega_{0}$ is the Kähler form of $M_{0}$ and $\left[\omega_{0}\right]$ is its de Rham cohomology class.

If we let $G_{\Lambda}$ denote the stabilizer of $\Lambda$ under the adjoint action, then

$$
L^{k} \cong\left(\pi^{*} L_{0}^{k}\right) \otimes \mathcal{L}_{-\lambda}
$$

where $\mathcal{L}_{-\lambda}$ is the complex line bundle associated with the principal $G_{\Lambda^{-}}$bundle

$$
p_{\Lambda}: \mu^{-1}(\Lambda) \rightarrow \mu^{-1}(\Lambda) / G_{\Lambda} \cong M_{\Lambda}
$$

and with the complex representation of $G_{\Lambda}$ of dimension 1 and weight $-\lambda$. This is true because the first Chern class of $L_{k}$ is equal to $L=\pi^{*}\left[\omega_{0}\right]+\left[\widetilde{\Omega}_{\Lambda}\right]$, where $\widetilde{\Omega}_{\Lambda}$ is a form on $M_{\Lambda}$ which, when restricted to a fiber, is the Kirillov-Kostant-Souriau form $\Omega_{\Lambda}$ on the coadjoint orbit $\mathcal{O}_{\Lambda}$. Furthermore,

$$
\mu^{-1}(\Lambda) / G_{\Lambda} \cong \mu^{-1}(0) / G_{\Lambda}
$$

for $\Lambda$ close to 0 . This yields the fibration $\pi$ given in (2). By the Borel-Weil-Bott theorem 3], the pushforward of $\mathcal{L}_{-\lambda}$ under this fibration is the vector bundle $\mathcal{V}(\lambda)^{*}$, and all higher direct images vanish.

By the Grothendieck-Riemann-Roch theorem [10], we have

$$
R R\left(M_{\Lambda}, L^{k}\right)=R R\left(M_{0}, L_{0}^{k} \otimes \mathcal{V}(\lambda)^{*}\right)
$$

using (5) and the fact that higher direct images vanish.

2.2. The non-Kähler case. When $M_{0}$ and $M_{\Lambda}$ are not Kähler, we must rely on an explicit argument using the Chern character and the Todd class. By the Normal Form Theorem ([8], Theorem 39.3 and Prop. 40.1) we can write the symplectic form on $M_{\Lambda}$ as $\omega_{\Lambda}=\pi^{*} \omega_{0}+\widetilde{\Omega}_{\Lambda}$, where $\widetilde{\Omega}_{\Lambda}$ is a form on $M_{\Lambda}$ which, when restricted to a fiber, is the Kirillov-Kostant-Souriau form $\Omega_{\Lambda}$ on the coadjoint orbit $\mathcal{O}_{\Lambda}$. Thus

$$
\operatorname{ch}\left(L^{k}\right)=e^{k \omega_{\Lambda}}=e^{k \pi^{*} \omega_{0}} e^{k \widetilde{\Omega}_{\Lambda}} .
$$

Next, we can split the tangent bundle of $M_{\Lambda}$ as $T M_{\Lambda}=\pi^{*} T M_{0} \oplus \mathcal{T}$, where $\mathcal{T}$ is the vertical bundle whose fiber over a point $x \in M_{\Lambda}$ is the tangent space to the fiber of $\pi$ over $x$. Since the Todd class is multiplicative, $\operatorname{Td}\left(M_{\Lambda}\right)=\operatorname{Td}\left(T M_{\Lambda}\right)=\pi^{*} \operatorname{Td}\left(T M_{0}\right) \operatorname{Td}(\mathcal{T})$.

Combining with the expression for $\operatorname{ch}\left(L^{k}\right)$, we have

$$
R R\left(M_{\Lambda}, L^{k}\right)=\int_{M_{\Lambda}} \operatorname{ch}\left(L^{k}\right) \operatorname{Td}\left(M_{\Lambda}\right)=\int_{M_{\Lambda}} e^{k \pi^{*} \omega_{0}} \pi^{*} \operatorname{Td}\left(M_{0}\right) e^{k \widetilde{\Omega}_{\Lambda}} \operatorname{Td}(\mathcal{T}) .
$$

The product of the first two factors $e^{k \pi^{*} \omega_{0}} \pi^{*} \operatorname{Td}\left(M_{0}\right)$ is written in terms of objects defined solely on the base $M_{0}$, and so we turn our attention to the other factors $e^{k \widetilde{\Omega}_{\Lambda}} \operatorname{Td}(\mathcal{T})$. Our strategy will be to integrate over the fiber of $\pi$, and be left with an integral over only the 
base $M_{0}$. Now $\mathcal{T}$ is a bundle over $M_{\Lambda}$, and so $\operatorname{Td}(\mathcal{T}) \in H^{*}\left(M_{\Lambda}\right)$. If $\imath_{x}: \mathcal{O}_{\Lambda} \hookrightarrow M_{\Lambda}$ is the inclusion map from $\mathcal{O}_{\Lambda}$ to the fiber over $x \in M_{0}$, then $\imath_{x}^{*}(\mathcal{T}) \cong T \mathcal{O}_{\Lambda}$.

Suppose $M$ is equipped with a complex vector bundle $\mathcal{V}$ (with fiber $\mathfrak{t} \otimes \mathbb{C}$ ) with an action of $G$ compatible with the action on $M$. Then $\mathcal{V}$ descends to a vector bundle $E$ on $M_{0}$. The characteristic classes of $E$ come from the invariant polynomials on $\mathfrak{g}$ via the Kirwan map. (The Kirwan map $\kappa: H_{G}^{*}(M) \rightarrow H^{*}\left(M_{0}\right)$ is the composition of the restriction map $r: H_{G}^{*}(M) \rightarrow H_{G}^{*}\left(\mu^{-1}(0)\right)$ with the isomorphism $H_{G}^{*}\left(\mu^{-1}(0)\right) \cong H^{*}\left(\mu^{-1}(0) / G\right)$, which is valid when 0 is a regular value for $\mu$. See [12.) We assume cohomology with rational, real or complex coefficients.

We assume the vector bundle $E$ over $M_{0}$ has the property that its pullback to $M_{\Lambda}$ splits as the direct sum of a collection of line bundles $L_{i}$ (in other words $M_{\Lambda}$ is a splitting manifold for $E$ over $\left.M_{0}\right)$. We then define $e_{i} \in H^{2}\left(M_{\Lambda}\right)$ by $e_{i}=c_{1}\left(L_{i}\right)$. The characteristic class of $E$ associated to an invariant polynomial $\tau \in S\left(\mathfrak{t}^{*}\right)^{W}$ is then given by

$$
c_{\tau}(E)=\tau\left(e_{1}, \ldots, e_{\ell}\right)
$$

(where $\ell$ is the rank of $T$ ). For example, if $G=U(n)$ the invariant polynomials are generated by the elementary symmetric polynomials [19]. The motivating example (the case treated in [13]) is the case where $M_{0}$ is the moduli space $M(n, d)$ of semistable holomorphic vector bundles of rank $n$ and degree $d$ over a Riemann surface (when $n$ and $d$ are two coprime positive integers), and $M_{\Lambda}$ is the corresponding moduli space of parabolic bundles. In this case the vector bundle $E$ is the universal bundle (see [1]).

By Section 14 in [1],

$$
\operatorname{Td}\left(\mathcal{O}_{\Lambda}\right)=\prod_{\gamma>0} \frac{\gamma(\mathcal{E})}{1-e^{-\gamma(\mathcal{E})}}=\prod_{\gamma>0} \frac{\gamma(\mathcal{E}) e^{\frac{1}{2} \gamma(\mathcal{E})}}{\left(e^{\frac{1}{2} \gamma(\mathcal{E})}-e^{-\frac{1}{2} \gamma(\mathcal{E})}\right)}
$$

where $\gamma$ are the roots of $G$, and $\mathcal{E}=\left(e_{1}, \ldots, e_{\ell}\right) \in H^{2}\left(M_{\Lambda}\right) \otimes \mathbb{R}^{\ell}$.

For example, if $G=U(n)$, under these hypotheses we obtain that the $j$-th Chern class is

$$
c_{j}(E)=\kappa\left(\left\{\tau_{j}\right\}\right)
$$

where $\tau_{j}$ (the $j$-th elementary symmetric polynomial) is regarded as an element of $H_{G}^{*}(\mathrm{pt})=S\left(\mathfrak{g}^{*}\right)^{G}$ and $\kappa: H_{G}^{*}(M) \rightarrow H^{*}\left(M_{0}\right)$ is the Kirwan map.

We introduce a basis $\hat{u}_{i}, i=1, \ldots, \ell$ for the integer lattice $\Lambda^{I}$ of $G$ (where $\ell$ is the rank of $G$ ). This enables us to define elements $e_{j} \in H^{2}\left(M_{\Lambda}\right), j=1, \ldots, \ell$ satisfying $c_{1}\left(L_{j}\right)=e_{j}$, where $e_{j}$ restricts on the fibers of $\pi$ to the generator $\alpha_{j}$ (for $j=1, \ldots, \ell$ ) of $H^{2}(G / T, \mathbb{Z}) \cong H^{1}(T, \mathbb{Z})$ corresponding to the $j$-th fundamental weight of $G$ (an element of $\left.\left.\operatorname{Hom}(T, U(1)) \cong H^{1}(T, \mathbb{Z})\right)\right)$. Using this notation, we have

Lemma 2.1. Let $\Lambda=\sum_{i=1}^{\ell} \Lambda_{i} \hat{u}_{i}$. Then the standard Kirillov-Kostant symplectic form $\Omega_{\Lambda}$ on $\mathcal{O}_{\Lambda}$ is given by

$$
\Omega_{\Lambda}=\sum_{j=1}^{\ell} \Lambda_{j} \alpha_{j}
$$

where the $\hat{u}_{i}$ and $\alpha_{j}$ are as defined above. 
Proof: This is a standard result (see for instance [2], Lemma 7.22).

The roots $\gamma$ also lie in the weight lattice $\Lambda^{W}$. Writing the pairing of $\mathfrak{t}^{*}$ and $\mathfrak{t}$ as as $(\cdot, \cdot)$ we have (still when restricted to the fiber)

$$
\begin{aligned}
e^{k \widetilde{\Omega}_{\Lambda}} \operatorname{Td}\left(\mathcal{O}_{\Lambda}\right) & =e^{\sum \lambda_{i} e_{i}} \prod_{\gamma>0} e^{\frac{1}{2}(\gamma, \mathcal{E})}\left[\prod_{\gamma>0} \frac{(\gamma, \mathcal{E})}{\left(e^{\frac{1}{2}(\gamma, \mathcal{E})}-e^{-\frac{1}{2}(\gamma, \mathcal{E})}\right)}\right] \\
& =e^{(\lambda, \mathcal{E})} e^{\frac{1}{2} \sum_{\gamma>0}(\gamma, \mathcal{E})} \prod_{\gamma>0} \frac{(\gamma, \mathcal{E})}{\left(e^{\frac{1}{2}(\gamma, \mathcal{E})}-e^{-\frac{1}{2}(\gamma, \mathcal{E})}\right)} \\
& =e^{(\lambda+\rho, \mathcal{E})} \prod_{\gamma>0} \frac{(\gamma, \mathcal{E})}{\left(e^{\frac{1}{2}(\gamma, \mathcal{E})}-e^{-\frac{1}{2}(\gamma, \mathcal{E})}\right)}
\end{aligned}
$$

where $\rho$ is half the sum of the positive roots. Notice that

$$
e^{k \widetilde{\Omega}_{\Lambda}} \operatorname{Td}\left(\mathcal{O}_{\Lambda}\right)\left[\mathcal{O}_{\Lambda}\right]=R R\left(\mathcal{O}_{\Lambda}, L^{k}\right)
$$

which equals $\operatorname{dim} V_{\lambda}$ by the Bott-Borel-Weil theorem (see [3] or [20]), where $V_{\lambda}$ is a representation of $G$ with highest weight $\lambda$ (we have assumed that $\lambda$ is in the fundamental Weyl chamber).

After evaluating on the fundamental cycle of $M_{\Lambda}$, the equation (8) equals

$$
\frac{1}{|W|}\left(\sum_{\sigma \in W}(-1)^{\sigma} \frac{e^{(\sigma(\lambda+\rho), \mathcal{E})}}{\prod_{\gamma>0}\left(e^{\frac{1}{2}(\gamma, \mathcal{E})}-e^{-\frac{1}{2}(\gamma, \mathcal{E})}\right)}\right) \prod_{\gamma>0}(\gamma, \mathcal{E})\left[M_{\Lambda}\right] .
$$

The expression in brackets in (10) is unchanged under the action of the Weyl group $\mathcal{E} \mapsto w \mathcal{E}$.

Let $\left\{\tau_{r}\right\}(r=1, \ldots, n(G))$ be a set of generators for the ring $S\left(\mathfrak{t}^{*}\right)^{W}$ of Weyl invariant polynomials on $\mathfrak{t}$, where $n(G)$ is the number of generators. By Proposition 3.6 in [13], $\left.\tau_{r}\left(e_{1}, \ldots, e_{\ell}\right)\right)=\pi^{*} a_{r}$ for $a_{r}$ a class on $M_{0}$. Therefore the factor (10) can be written as a function of the invariant polynomials $\tau_{r}$ applied to the $e_{j}$ 's,

$$
\mathcal{S}_{\lambda}\left(\tau_{r}\left(e_{1}, \ldots, e_{\ell}\right)\right) \stackrel{\text { def }}{=} \sum_{\sigma \in W}(-1)^{\sigma} \frac{e^{(\sigma(\lambda+\rho), \mathcal{E})}}{\prod_{\gamma>0}\left(e^{\frac{1}{2}(\gamma, \mathcal{E})}-e^{-\frac{1}{2}(\gamma, \mathcal{E})}\right)}
$$

We see that $\mathcal{S}_{\lambda}$ is actually a polynomial in the $e_{j}$. Because $e_{j}^{N+1}=0$ where $2 N=$ $\operatorname{dim}_{\mathbb{R}} M_{\Lambda}$, it follows immediately that after evaluating on the fundamental class of $M_{0}$,

Theorem 2.2. $\mathcal{S}_{\lambda}$ is a polynomial in $\lambda$ of degree $\leq N$.

We can replace the term $e^{k \tilde{\Omega}_{\Lambda}} \operatorname{Td}(V)$ in the integral with

$$
\frac{1}{|W|} \mathcal{S}_{\lambda}\left(\pi^{*} a_{1}, \ldots, \pi^{*} a_{n(G)}\right) \prod_{\gamma>0}(\gamma, \mathcal{E})
$$

to get

$$
R R\left(M_{\Lambda}, L^{k}\right)=\frac{1}{|W|} \int_{M_{\Lambda}} e^{k \pi^{*} \omega_{0}} \pi^{*} \operatorname{Td}\left(M_{0}\right) \mathcal{S}_{\lambda}\left(\pi^{*} a_{1}, \ldots, \pi^{*} a_{n(G)}\right) \prod_{\gamma>0}(\gamma, \mathcal{E}) .
$$


All of the factors in the integral except for $\prod(\gamma, \mathcal{E})$ are constant on each fiber, and so we have

$$
R R\left(M_{\Lambda}, L^{k}\right)=\frac{1}{|W|} \int_{M_{0}} e^{k \omega_{0}} \operatorname{Td}\left(M_{0}\right) \mathcal{S}_{\lambda}\left(a_{1}, \ldots, a_{n(G)}\right) \int_{\mathcal{O}_{\Lambda}} \prod_{\gamma>0}(\gamma, \mathcal{E}) .
$$

Since

we have finally

$$
\int_{\mathcal{O}_{\Lambda}} \prod_{\gamma>0}(\gamma, \mathcal{E})=|W|
$$

Theorem 2.3. The Riemann-Roch number of a symplectic fibration $M_{\Lambda}$ is given by

$$
R R\left(M_{\Lambda}, L^{k}\right)=\int_{M_{0}} e^{k \omega_{0}} \operatorname{Td}\left(M_{0}\right) \mathcal{S}_{\lambda}\left(a_{1}, \ldots, a_{n(G)}\right) .
$$

where $\mathcal{S}_{\lambda}$ is defined at (11).

Theorem 2.4. When $\Lambda \in \Lambda^{W}$ is a weight in the fundamental Weyl chamber, then the limit as $k \rightarrow \infty$ of

$$
\frac{R R\left(M_{\Lambda}, L^{k}\right)}{k^{N}}
$$

(where as above $2 N=\operatorname{dim}_{\mathbb{R}}\left(M_{\Lambda}\right)$ ) is $\operatorname{vol} M_{0} \operatorname{dim} V_{\Lambda-\rho}$.

Proof: This limit is given by vol $M_{\Lambda}$. The symplectic volume is given by $\operatorname{vol} M_{0} \operatorname{vol} \mathcal{O}_{\Lambda}$. The symplectic volume of $\mathcal{O}_{\Lambda}$ is given at [2] (Proposition 7.26) as

$$
\operatorname{vol}\left(\mathcal{O}_{\Lambda}\right)=\frac{\prod_{\alpha>0}<\alpha, \Lambda>}{\prod_{\alpha>0}<\alpha, \rho>} .
$$

This gives the value of $\operatorname{dim} V_{\Lambda-\rho}$, using the Weyl dimension formula [6].

Proposition 2.5. Let $\lambda \in \Lambda^{W}$ be a weight in the fundamental Weyl chamber, and define $\Lambda(k)=\lambda / k$. Let $N_{0}=\frac{1}{2} \operatorname{dim} M_{0}$. Then

$$
\lim _{k \rightarrow \infty} \frac{1}{k^{N_{0}}} R R\left(M_{\Lambda}, L^{k}\right)=\left(\operatorname{vol} M_{0}\right)\left(\operatorname{dim} V_{\lambda-\rho}\right) .
$$

Proof: For $X \in \mathbf{t}$ we introduce

$$
S_{\lambda}(X) \stackrel{\text { def }}{=} \sum_{\sigma \in W}(-1)^{\sigma} \frac{e^{(\sigma(\lambda+\rho), X)}}{\prod_{\gamma>0}\left(e^{\frac{1}{2}(\gamma, X)}-e^{-\frac{1}{2}(\gamma, X)}\right)}
$$

Notice that we are fixing $\lambda$ and allowing $\Lambda=\lambda / k$ to vary as $k$ varies. Notice that by the Weyl character formula ([20], Proposition 14.2.2) we have

$$
S_{\lambda}(X)=\chi_{\lambda}(\exp X)
$$

where $\chi_{\lambda}$ is the character of the representation with lowest weight $-\lambda$. Theorem 2.3 gives the result, noting that in the limit $k \rightarrow \infty$ the leading order term in $k$ comes by integrating $\left(k \omega_{0}\right)^{N_{0}}$ so the factor $\mathcal{S}_{\lambda}\left(a_{1}, \ldots, a_{n(G)}\right)$ contributes only its value when all the arguments $a_{i}$ are replaced by 0 , in other words when the argument of $S_{\lambda}(X)$ is replaced by $X=0$. This is the value $\chi_{\lambda}(0)$, in other words the dimension of $V_{\lambda-\rho}$. 
Example 2.6. When $G=S U(2)$, the value of $\mathcal{S}_{\lambda}$ is (recalling that $\lambda$ is a positive integer)

$$
\mathcal{S}_{\lambda}\left(a_{2}\right)=\kappa\left(S_{\lambda}(X)\right)
$$

where

$$
\begin{gathered}
S_{\lambda}(X)=\frac{1}{2} \frac{e^{(\lambda+1) X}-e^{-(\lambda+1) X}}{e^{X}-e^{-X}} \\
=\frac{1}{2}(\cosh (X)+\cdots+\cosh (\lambda-1) X+\cosh \lambda X) .
\end{gathered}
$$

Here we have introduced a formal variable $X$ for which $\kappa\left(X^{2}\right)=a_{2} \in H^{4}\left(M_{0}\right)$. It follows that $\mathcal{S}_{\lambda}$ is a polynomial in $\lambda$ of order $N+1$, because the terms which contribute from the Taylor expansion of order $X^{N}$ are $\sum_{j=1}^{\lambda} j^{N}$ which is of order $\lambda^{N+1}$. Because we are integrating over $M_{\Lambda}$, the ring of polynomials in the variable $X$ gets truncated by imposing the relation $X^{N+1}=0$. In this example $S_{\lambda}(0)=\lambda / 2$.

\section{ThE JEFFREY-KIRWAN RESIDUE FORMULA}

In the final two sections of this paper, we express the Riemann-Roch number on a symplectic fibration in terms of data at the fixed point set of the maximal torus $T$ of $G$ (assuming $\Lambda$ is generic so its stabilizer under the coadjoint action is $T$ ). In this way, we obtain a second proof of Theorem 2.3.

The residue formula 14 expresses cohomology pairings on reduced spaces $M_{0}$ in terms of a multi-dimensional residue of certain rational holomorphic functions on $\mathfrak{t}$ 14. This formula is valid provided 0 is a regular value of the moment map. The cohomology classes $\beta_{0}$ on $M_{0}$ are assumed to come from equivariant cohomology classes $\beta$ on $M$ via the Kirwan map [12]. The fixed point data are

- the value of the moment map at a component $F$ of the fixed point set of the maximal torus $T$

- the restriction of $\beta$ to the $F$

- the equivariant Euler class $e_{F}$ of the normal bundle to $F$ (which involves the weights of the action of $T$ on the normal bundle, as well as the ordinary Chern roots of the normal bundle).

The residue formula takes the form

$$
\int_{M_{0}} e^{\omega_{0}} \beta_{0}=C \operatorname{Res}\left(\sum_{F} \int_{F} \frac{e^{\omega+\mu(F)(X)} \beta(X)}{e_{F}(X)}\right)
$$

where $C$ is a nonzero constant, $X \in \mathfrak{t} \otimes \mathbb{C}$ is a formal variable (in the Cartan model for equivariant cohomology), and Res is defined at (19) below.

We can readily identify the equivariant cohomology class giving rise to the RiemannRoch number of a prequantum line bundle. The class $e^{\omega_{0}}$ is the Chern character of the line bundle over $M_{0}$, while $e^{\omega+\mu(F)(\cdot)}$ is the equivariant Chern character of the line bundle over $M$. The relevant class $\beta_{0}$ is the Todd class of $M_{0}$, which arises in the image of the Kirwan map using the equivariant Todd class $\operatorname{Td}_{G}$ (see [16] and Proposition 4.1 below). The residue formula has been applied to studying Riemann-Roch numbers in [15] and 
[16. Here we study the residue formula for the Riemann-Roch number on a symplectic fibration.

The residue formula applies to compact $M$ reduced by any compact group $G$. The computation of terms in the residue formula depends on the choice of a cone $\Gamma$ in $\mathfrak{t}$, even though the result of the formula is independent of this choice. Let $\gamma_{1}, \ldots, \gamma_{k}$ be the set of all weights that occur by the $T$ action at any of the fixed point components. Choose some $\xi \in \mathbf{t}$ such that $\gamma_{i}(\xi) \neq 0$ for all $i$. Let $\beta_{i}=\gamma_{i}$ if $\gamma_{i}(\xi)>0$ and $\beta_{i}=-\gamma_{i}$ if $\gamma_{i}(\xi)<0$. Thus $\beta_{i}(\xi)>0$ for all $i$. The cone $\Gamma$ is the set of all vectors in $\mathfrak{t}$ which behave like $\xi$ :

$$
\Gamma=\left\{X \in \mathfrak{t}: \beta_{i}(X)>0, \text { for all } i\right\} .
$$

Theorem 3.1 (Jeffrey-Kirwan). Let $(M, \omega)$ be a compact symplectic manifold with a Hamiltonian $T$ action and moment map $\Phi$, where $T$ is a compact torus. Denote by $\mathcal{F}$ the connected components of the fixed point set of $T$ on $M$. Let $p$ be a regular value of $\Phi$ and $\omega_{p}$ the Marsden-Weinstein reduced symplectic form on $M_{p}$. Then for $\beta \in H_{T}^{*}(M)$ and $\kappa_{p}: H_{T}^{*}(M) \rightarrow H^{*}\left(M_{p}\right)$ we have

$$
\int_{M_{p}} \kappa_{p}(\beta) e^{\omega_{p}}=C \cdot \operatorname{res}^{\Gamma}\left(\sum_{F \in \mathcal{F}} e^{i(\Phi(F)-p)(X)} \int_{F} \frac{\iota_{F}^{*}\left(\beta(X) e^{\omega}\right)}{e_{F}(X)}[d X]\right)
$$

where $C$ is a non-zero constant, $X$ is a variable in $\mathfrak{t} \otimes \mathbb{C}$, and $e_{F}(X)$ is the equivariant Euler class of the normal bundle to $F$ in $M$. The multi-dimensional residue $\operatorname{res}^{\Gamma}$ is defined below at (21).

The residue can be defined as follows (see [16] Proposition 3.4). For $f$ a meromorphic function of one complex variable $z$ which is of the form $f(z)=g(z) e^{i \lambda z}$ where $g$ is a rational function, we define

$$
\operatorname{res}_{z}^{+} f(z) d z=\sum_{b \in \mathbb{C}} \operatorname{res}\left(g(z) e^{i \lambda z} ; z=b\right) .
$$

We extend this definition by linearity to linear combinations of functions of this form.

Viewing $f$ as a meromorphic function on the Riemann sphere and observing that the sum of all the residues of a meromorphic 1-form on the Riemann sphere is 0 , we observe that

If $X \in \mathfrak{t}$, define

$$
\operatorname{res}_{z}^{+}(f(z) d z)=-\operatorname{Res}_{z=\infty}(f(z) d z) .
$$

$$
h(X)=\frac{q(X) e^{i \lambda(X)}}{\prod_{j=1}^{k} \beta_{j}(X)}
$$

for some polynomial function $q(X)$ of $X$ and some $\lambda, \beta_{1}, \ldots, \beta_{k} \in \mathfrak{t}^{*}$. Suppose that $\lambda$ is not in any proper subspace of $\mathfrak{t}^{*}$ spanned by a subset of $\left\{\beta_{1}, \ldots, \beta_{k}\right\}$. Let $\Gamma$ be any nonempty open cone in $\mathfrak{t}$ contained in some connected component of

$$
\left\{X \in \mathfrak{t}: \beta_{j}(X) \neq 0,1 \leq j \leq k\right\}
$$

Then for a generic choice of coordinate system $X=\left(X_{1}, \ldots, X_{l}\right)$ on $\mathfrak{t}$ for which $(0, \ldots, 0,1) \in$ $\Gamma$ we have

$$
\operatorname{res}^{\Gamma}(h(X)[d X])=\operatorname{Jacres}_{X_{1}}^{+} \circ \ldots \circ \operatorname{res}_{X_{l}}^{+}\left(h(X) d X_{1} \ldots d X_{l}\right)
$$


where the variables $X_{1}, \ldots, X_{m-1}$ are held constant while calculating res $_{X_{m}}^{+}$, and Jac is the determinant of any $l \times l$ matrix whose columns are the coordinates of an orthonormal basis of $\mathfrak{t}$ defining the same orientation as the chosen coordinate system. We assume that if $\left(X_{1}, \ldots, X_{l}\right)$ is a coordinate system for $X \in \mathfrak{t}$, then $(0,0, \ldots, 1) \in \Gamma$. We also require an additional technical condition on the coordinate systems, which is valid for almost any choice of coordinate system (see Remark 3.5 (1) from [16]).

\section{FiXED POINT FORMULAS}

We wish to use the residue formula to calculate the integral in (13). In (20) we are interested in $\beta(X)=\operatorname{Td}_{G}\left(M \times \mathcal{O}_{\Lambda}\right) \operatorname{Td}_{G}^{-1}\left(\mathfrak{g}_{\mathrm{ad}} \oplus \mathfrak{g}_{\mathrm{ad}}^{*}\right)$ which maps to $\operatorname{Td}\left(M_{\Lambda}\right)$ under the Kirwan map.

The components of the fixed point set for the action of $T$ on $M \times \mathcal{O}_{\Lambda}$ are of the form $F \times\{\sigma \Lambda\}$ where $F$ is a component of the $T$ fixed point set of $M$ and $\sigma \in W$. The equivariant Euler class at this fixed point is $e_{F}(X)(-1)^{\sigma} \prod_{\gamma>0} \gamma(X)$. Thus the residue formula becomes

$$
\begin{gathered}
\int_{M_{\Lambda}} e^{k \omega_{\Lambda}} \operatorname{Td}\left(M_{\Lambda}\right)=C \operatorname{Res}\left(\sum_{\sigma \in W} \sum_{F \in \mathcal{F}}(-1)^{\sigma} e^{\sigma \lambda(X)} e^{k \mu_{F}(X)} \times\right. \\
\left.\times \frac{\mathcal{D}^{2}(X)}{\prod_{\gamma>0} \gamma(X) \operatorname{Td}_{G}\left(\mathfrak{g}_{\mathrm{ad}} \oplus \mathfrak{g}_{\mathrm{ad}}^{*}\right)} \int_{F \times\{\sigma \Lambda\}} \frac{e^{k \omega}}{e_{F}(X)} \operatorname{Td}_{G}(M) \operatorname{Td}_{G}\left(\mathcal{O}_{\Lambda}\right)\right) .
\end{gathered}
$$

Here, $\omega$ is the symplectic form on $F, C$ is an overall constant given in the statement of Theorem 3.1. $\mathcal{D}^{2}(X)$ is the product of all the roots of $\mathfrak{g}$, and $e_{F}(X) \in H_{T}^{*}(M)$ is the equivariant Euler class of the normal bundle to $F$. The $F$ are components of the fixed point set $\mathcal{F}$ for the action of $T$ on $M$. We use Proposition 4.1 below for the Todd class.

Proposition 4.1. (15), Proposition 2.1) The formal equivariant cohomology class

$$
\operatorname{Td}_{G}(M) \operatorname{Td}_{G}^{-1}\left(\mathfrak{g}_{a d} \oplus \mathfrak{g}_{a d}^{*}\right)
$$

maps to $\operatorname{Td}\left(M_{0}\right)$ under $\kappa$, where $\mathfrak{g}_{a d}$ (resp. $\mathfrak{g}_{a d}^{*}$ ) denotes the product bundle $M \times \mathfrak{g}$ (resp. $\left.M \times \mathfrak{g}^{*}\right)$ with $G$ acting on $\mathfrak{g}$ by the adjoint action (resp. the coadjoint action).

Proof: We observe that $\kappa\left(\operatorname{Td}_{G}(M) \operatorname{Td}_{G}^{-1}\left(\mathfrak{g}_{\mathrm{ad}} \oplus \mathfrak{g}_{\mathrm{ad}}^{*}\right)\right)=\operatorname{Td}\left(M_{0}\right)$.

Proposition 4.2. The formal equivariant cohomology class $S_{\lambda}(X)$ maps to $\mathcal{S}_{\lambda}\left(a_{1}, \ldots, a_{n(G)}\right)$ under $\kappa$, where

$$
S_{\lambda}(X)=\frac{\sum_{\sigma \in W}(-1)^{\sigma} e^{(\sigma(\lambda+\rho), X)}}{\prod_{\gamma>0}\left(e^{\frac{1}{2}(\gamma, X)}-e^{-\frac{1}{2}(\gamma, X)}\right)} .
$$

Proof: The expression defining $S_{\lambda}$ is the same as the expression (11) defining $\mathcal{S}_{\lambda}$, with the expression $\mathcal{E}$ replaced by the variable $X \in \mathfrak{t}$. Thus $S_{\lambda} \in S\left(\mathfrak{t}^{*}\right)^{W}$ can be viewed as an equivariant cohomology class on $M$. 
Since $S_{\lambda}$ is symmetric under the action of the Weyl group, it is a function of the invariant polynomials $\tau_{r}(X)$. Since $\kappa\left(\tau_{r}\right)=a_{r}$ by definition of the $a_{r}$, when $\kappa$ is applied to $S_{\lambda}$, the result will be $\mathcal{S}_{\lambda}$. Since $\kappa$ is a ring homomorphism, the result follows.

The expression (22) is equal to the expression (10) which we derived in Section 2 for

$$
\int_{M_{0}} e^{k \omega_{0}} \operatorname{Td}\left(M_{0}\right) \mathcal{S}_{\lambda}\left(a_{1}, \ldots, a_{n(G)}\right) .
$$

To see this, we only need to evaluate (10) using the residue formula, and use the fact that

$$
\left.\frac{1}{\prod_{\gamma>0} \gamma(X)} \operatorname{Td}_{G}\left(\mathcal{O}_{\Lambda}\right)\right|_{F \times\{\sigma \Lambda\}}=(-1)^{\sigma} \prod_{\gamma>0} \frac{1}{1-e^{-\sigma \gamma(X)}}
$$

(which appears in (22)) is the same as

$$
\frac{e^{\sigma \rho}}{\prod_{\gamma>0}\left(e^{\gamma / 2}-e^{-\gamma / 2}\right)}
$$

(which appears when we evaluate (10) using the residue formula). Hence (25) becomes

$$
C \operatorname{res}\left(\mathcal{D}^{2}(X) S_{\lambda}(X) \sum_{F \in \mathcal{F}} e^{i \mu_{T}(F)(X)} \int_{F} \frac{\imath_{F}^{*}\left(\operatorname{Td}_{G}(M) \operatorname{Td}_{G}^{-1}\left(\mathfrak{g}_{\mathrm{ad}} \oplus \mathfrak{g}_{\mathrm{ad}}^{*}\right) e^{k \omega}\right)}{e_{F}(X)}[d X]\right),
$$

which is the same as (22). Thus we have obtained a second proof of Theorem 2.3 .

\section{REFERENCES}

[1] M.F. Atiyah, R. Bott, The Yang-Mills equations over Riemann surfaces, Phil. Trans. Roy. Soc. Lond. A308 (1982) 523-615.

[2] N. Berline, E. Getzler, M. Vergne, Heat Kernels and Dirac Operators, Springer-Verlag (Grundlehren vol. 298), 1992.

[3] R. Bott, Homogeneous vector bundles. Annals of Math. 66 (1957) 203-248.

[4] M. Brion, Cohomologie équivariante des points semi-stables, J. reine angew. Math., Vol. 421 (1991), 125-140.

[5] J.J. Duistermaat, The heat kernel Lefschetz fixed point formula for the Spin-C Dirac operator, Birkhäuser, 1996.

[6] J.J. Duistermaat, J.A.C. Kolk, Lie Groups, Springer-Verlag, 2002.

[7] V. Guillemin, Reduced phase spaces and Riemann-Roch, in Lie Groups and Geometry (Proceedings in honor of B. Kostant), R. Brylinski et al., eds., Progress in Mathematics 123, Birkhäuser, 1995, 305-334.

[8] V. Guillemin, S. Sternberg, Symplectic techniques in physics, Cambridge University Press, 1984.

[9] V. Guillemin, E. Lerman, S. Sternberg On the Kostant multiplicity formula, J. Geom. Phys. 5 (1988) $721-750$.

[10] R. Hartshorne, Algebraic Geometry, Springer-Verlag (Graduate Texts in Mathematics 52), 1977.

[11] F. Hirzebruch, Topological methods in algebraic geometry, 3rd edition, Springer, 1995.

[12] F. Kirwan, Cohomology of quotients in symplectic and algebraic geometry, Princeton University Press, 1984.

[13] L.C. Jeffrey, The Verlinde formula for parabolic bundles. J. London Math. Soc., 63 (2001), 754-768.

[14] L.C. Jeffrey, F.C. Kirwan, Localization for nonabelian group actions. Topology 34 (1995), 291-327.

[15] L.C. Jeffrey, F.C. Kirwan, On localization and Riemann-Roch numbers for symplectic quotients. Quart. J. Math. 47 (1996), 165-186. 
[16] L. C. Jeffrey, F. C. Kirwan, Localization and the quantization conjecture, Topology 36 (1997), 647-693.

[17] E. Meinrenken, On Riemann-Roch formulas for multiplicities. Journal of the American Mathematical Society 9 (1996), 373-390.

[18] E. Meinrenken, Symplectic surgery and the Spin-c Dirac operator. Advances in Mathematics 134 (1998), 240-277.

[19] J. Milnor, J. Stasheff, Characteristic Classes. Princeton University Press (Annals of Mathematics Studies vol. 76), 1974.

[20] A. Pressley, G. B. Segal, Loop Groups, Oxford University Press, 1988.

[21] M. Vergne, Multiplicities formula for geometric quantization Part I, Duke Math. J. 82 (1996) 143179; Multiplicities formula for geometric quantization Part II, Duke Math. J. 82 (1996) 181-194.

University of Toronto, Toronto, On, Canada

E-mail address: umbra@math.toronto.edu

University of Toronto, Toronto, On, Canada

E-mail address: jeffrey@math.toronto.edu 